Problemy sovremennogo pedagogicheskogo obrazovaniya [Problems of modern teacher education], 69, 232-236. (In Russ., abstract in Eng.)

13. Volkov, Yu. A., Starostina, S. A. (2021). Aktivizaciya uchebno-poznavatel'noj deyatel'nosti s pomoshch'yu metoda «mozgovogo shturma» [Activation of educational and cognitive activity using the method of "brainstorming"]. In: Innovative technologies in higher school pedagogy. Materials of the VII International Interuniversity scientific and methodological conference (pp. 255-258). Sankt-Petersburg, Peterhof. (In Russ.)

14. Voronko, T. A. (2007). Zadachi kak sredstvo formirovaniya opyta issledovatel'skoj deyatel'nosti uchashchihsya Tasks as a means of forming the experience of students 'research activities [Tasks as a means of forming the experience of students 'research activities]. Nauka $i$ shkola [Science and school], 5, 47-49. (In Russ., abstract in Eng.)

15. Yaroshenko, S. N. (2007). Ponyatie «Aktivizaciya uchebno-poznavatel'noj deyatel'nosti» uchashchihsya $\mathrm{v}$ nauchno-pedagogicheskih issledovaniyah [The concept of "Activation of educational and cognitive activity" of students in scientific and pedagogical research]. Vestnik $O G U$ [OSU Bulletin], 9, 81-82. (In Russ., abstract in Eng.).

DOI: $10.24888 / 2500-1957-2021-3-20-29$

\begin{tabular}{c|l} 
УДК & ЦЕЛИ И ФОРМЫ ОРГАНИЗАЦИИ САМОСТОЯТЕЛЬНОЙ \\
378 & РАБОТЫ БАКАЛАВРОВ ЭКОНОМИКИ В СИСТЕМЕ \\
& ПРИКЛАДНОЙ МАТЕМАТИЧЕСКОЙ ПОДГОТОВКИ
\end{tabular}

Власов Дмитрий Анатольевич $\mid$ Российский экономический университет

к.П.н., доцент

им. Г.В. Плеханова

DAV495@gmail.com

г. Москва

\begin{abstract}
Аннотация. В центре внимания статьи цели и формы организации самостоятельной работы как компоненты методической системы прикладной математической подготовки бакалавров экономики. Представлены шесть целей самостоятельной работы студентов экономического бакалавриата, позволяющие акцентировать внимание на развитие инновационных компонентов профессиональной деятельности будущего экономиста в условиях цифровизации высшего экономического образования и необходимости реализации прикладного усиления математической подготовки. Особое внимание уделяется перечислению и содержательному описанию форм самостоятельной работы студентов экономического бакалавриата без непосредственного участия преподавателей математических дисциплин. Представленные в статье организационно-методические особенности самостоятельной работы бакалавров экономики позволяют развивать систему самостоятельной работы студентов, значимость которой в условиях реформирования высшей экономического образования возрастает.
\end{abstract}

Ключевые слова: бакалавр экономики, высшая экономическая школа, математическая подготовка, самостоятельная работа, форма, цель, цифровизация.

Новые условия, связанные с социально-экономическими преобразованиями, существенно изменили требования к качеству подготовки выпускников экономических университетов. Развитие инновационной экономики России невозможно без изменения роли 
самостоятельной работы студентов экономического бакалавриата, а также изменению подходов к её планированию и реализации в рамках учебного процесса в высшей экономической школе, частично проходящего в электронной образовательной среде университета. Активное внедрение цифровых технологий стимулирует необходимость обновления содержания, структуры и последовательности учебных дисциплин, обеспечения соответствия требованиям профессиональных и образовательных стандартов.

Реальные условия преподавательской деятельности в высшей экономической школе приводят к существенному росту ответственности членов профессорско-преподавательского состава за формирование и развитие компетенций, формируемых в процессе самостоятельной работы, за управление познавательными интересами студентов, которым должна быть предоставлена возможность выбора собственной образовательной траектории. Однако не менее важным нам представляется воспитание творческой активности будущих выпускников экономического университета, ответственного отношения к труду и инициативы. Процесс профессионального становления молодых специалистов, а также уровень их социальной востребованности во многом определяются умениями адаптировать уже известные приемы и методы к новым условиям, проявлением инициативы, навыками анализа ситуаций в различных информационных условиях, умениями решить нестандартные задачи [3], требующие привлечения дополнительной информации и учёта взаимодействий нескольких экономических агентов.

Способность к планированию и прогнозированию результатов хозяйственноэкономической деятельности зависит от уровня самостоятельности исследователей, от развитости компетенций по самостоятельному приобретению знаний из различных источников, оценке значимости и систематизации полученной информации, количественному анализу данных и прогнозированию развития экономических ситуаций на их основе, многосторонней и адекватной оценки взаимодействия и результатов деятельности экономических субъектов. Различные методические аспекты, связанные с управлением самостоятельной работой будущего экономиста, неоднократно были в центре внимания исследователей. Так, в работе [4] обоснована необходимость разработки специального педагогического сопровождения процесса формирования профессионально значимых качеств экономиста-менеджера в системе самостоятельной работы студентов. В публикации [7] уделяется внимание организационно-педагогическим условиям эффективности самостоятельной работы студентов-экономистов. В статье [8] указывается на связь вовлеченности в обучение студентов и качеством организации их самостоятельной работы, в публикации [5] организация самостоятельной работы студентов представляется важной проблемой преподавания математических дисциплин в реалиях цифрового образования.

Ранее в работах автора обоснована необходимость повышения качества прикладной математической подготовки будущего экономиста, представлены методические рекомендации по совершенствованию преподавания учебных дисциплин образовательной области «Математические методы в экономике», раскрыты возможности применения технологического подхода для развития содержания профессиональной подготовки будущего экономиста [1]. В рамках данной статьи будут рассмотрены цели и формы как компоненты системы самостоятельной работы студентов экономического бакалавриата по освоению экономико-математических методов.

Заметим, что к прикладным математическим дисциплинам, включенным в практику профессиональной подготовки будущего экономиста в экономическом университете, относятся «Методы оптимизации», «Теория игр», «Теория риска» [13], «Теория оптимального управления», «Вычислительная математика» [10], «Актуарная математика» [12], «Методы оптимальных решений» [6] и др. Несмотря на некоторую неоднородность содержания указанных учебных дисциплин, они связаны направленностью на развитие инновационных компонентов профессиональной компетентности выпускников экономических университетов, связанных с количественным обоснованием принимаемых решений, развитием модельных представлений о социально-экономических системах. Представим далее цели 
самостоятельной работы студентов экономического бакалавриата, поставленным нами в рамках прикладной математической подготовки, реализуемой в Российском экономическом университете им. Г. В. Плеханова и Финансовом университете при Правительстве Российской Федерации.

Цель 1. Систематизация и закрепление наиболее значимых элементов теоретических знаний и наиболее востребованных практических умений студентов экономического бакалавриата, относящихся к образовательной области «Математические методы в экономике».

Цель 2. Углубление и расширение теоретических знаний по количественным методам и математическому моделированию.

Цель 3. Формирование умений студентов экономического бакалавриата использовать новые циирровые инструментальные средства и базы данных для анализ соииальноэкономических ситуаций.

Цель 4. Поддержка прочесса поэтапного развития познавательных способностей и активности студентов экономического бакалавриата, относящихся к образовательной области «Математические методы в экономике».

Цель 5. Формирование самостоятельности математического мышления как компонента профессионального мышления, выраженных способностей к профессиональному саморазвитию в контексте применения количественных методов и математического моделирования, совершенствованию исследования социально-экономических ситуаций $u$ самоорганизации.

Цель 6. Развитие ключевых и профессиональных компетенций с акцентом на развитие исследовательских умений в области математических методов в экономике и анализе больших данных. Наиболее востребованными в данном контексте представляются исследования научной группы А. В. Хуторского, например [15].

Для развития системы управления учебной работой студентов экономическим университетов, реализуемой для прикладной математической подготовки будущего экономиста, нами выделены организационно-методические особенности использования различных форм самостоятельной работы студентов экономического бакалавриата без непосредственного участия преподавателей математических дисциплин. Считаем, то в процессе проектирования учебного процесса по математическим дисциплинам необходимо предусмотреть связи микроцелей, задающих учебный процесс на языке учебнопознавательной деятельности студентов экономического бакалавриата, с различными формами организации самостоятельной работы. Отметим, что вопросы технологического целеполагания и педагогического проектирования для преподавания математических дисциплин раскрыты в публикациях $[11,14]$.

Форма 1. Представление в виде иерархических структур (алгоритмов, фреймов и др.) и усвоение теоретического содержания конспектов основных учебных тем с использованием содержания лекционных занятий, а также дополнение их на основе рекомендованных цифровых ресурсов образовательного назначения и открытых источников информации. Moodle и корпоративная SLM, в которой к настоящему времени размещены цифровые образовательные ресурсы «Теория игр», «Теория риска», «Теория принятия решений» и др. Отметим, что рекомендации методически целесообразному использованию SLM Moodle в практике преподавания различных математических дисциплин представлены в работе [2].

Форма 2. Написание рефератов по основным темам образовательной области «Математические методы в экономике», подготовка к их публичной защите и аргументированной защите собственных позиций. Отражение в содержании рефератов необходимых теоретических аспектов, связанных с применением математических методов и количественного моделирования:

- теоретическое обоснование выбора внутримодельного исследования;

- доказательство сходимости метода;

- доказательство существования решения; 
- доказательство единственности найденного решения;

- оценка точности полученного решения в условиях невозможности реализации аналитического метода и применения численного метода и др.

- Отражение в содержании рефератов необходимых практических аспектов, связанных

- с границами применения изучаемых методов;

- возможными содержательными интерпретациями получаемых результатов в социально-экономических терминах;

- $\quad$ совершенствованием процесса принятия решений на основе количественных методов и математического моделирования;

- выработкой практических рекомендаций по использованию результатов математического моделирования в практике принятия решений в различных областях хозяйственно-экономической деятельности.

Заметим, что публичная защита рефератов является играет важную роль в пропедевтике последующей защиты выпускных квалификационных работ, а также в развитии практических навыков устной математической речи студентов, которой в современных условиях уделяется недостаточное внимание.

Форма 3. Подготовка студентов экономического бакалавриата к традиционным практическим занятиям по прикладным математическим дисциплинам и практическим занятиям в компьютерных классах, приближенных к будущей профессиональной деятельности благодаря интеграции цифровых и педагогических технологий. Основные механизмы интеграции цифровых и педагогических технологий в процессе прикладной математической подготовки позволяют по-новому организовать учебно-познавательную деятельность студентов, учитывая дидактический потенциал активно развивающихся цифровых технологий.

Форма 4. Работа над домашними заданиями в виде отдельных прикладных задач социально-экономической тематики, типовых расчетов с последовательным усложнением предлагаемых заданий, индивидуальных работ по отдельным разделам содержания прикладной математической подготовки и т. д. Заметим, то для обеспечения профессиональной направленности данной формы организации самостоятельной работы студентов экономического бакалавриата мы пришли к необходимости расширения перечня прикладных задач на применение различных математических методов и приемов анализа данных: среди которых задачи на прогнозирование развития социально-экономических ситуаций, анализ рисковых ситуаций, возникающих в различных областях хозяйственноэкономической деятельности, задачи на анализ взаимодействия нескольких экономических агентов с учётом выраженности антагонизма, задачи принятия решений в условиях полной и частичной неопределенности и др.

Форма 5. Прохождение студентами технологических диагностик по основным учебным темам, в том числе и с использованием для своевременного контроля самоконтроля уровня сформированности значений, умений и компетенций, относящихся к образовательной области «Математические методы в экономике». Важной методической особенностью составления банка технологических диагностик является обеспечение полного соответствия их содержания технологическому целеполаганию, осуществляемому преподавателем высшей школы.

Обратимся к результатам методического анализа учебной темы «Линейное программирование» для организации самостоятельной работы студентов экономического бакалавриата. Отметим, что данная учебная тема играет существенную роль в развитии модельных представлений будущих бакалавров экономики и для удобства управления системой самостоятельной работы студентов мы предлагаем разбить данную учебную тему на пять взаимосвязанных разделов.

Раздел 1. Математический аппарат для анализа соџиально-экономических ситуаций, приводящая к задачам линейного программирования. Самостоятельная работа студентов экономического бакалавриата по данному разделу направлена на обеспечение и 
профессионально ориентированного понимания математического аппарата линейного программирования. Действительно, в современных условиях математизации экономики и экономических исследований необходимо вооружить будущего бакалавра экономики не только к базовым математическим методам и моделями, но и сформировать навыки самостоятельной разработки экономико-математических приемов и методов, которые впоследствии выпускники экономического университета смогут использовать в профессиональной деятельности. Однако в условиях наблюдающийся тенденции сокращения часов на математическую подготовку в высшей экономической школе мы считаем необходимым предостеречь от излишней формализации и неоправданного усложнения математического аппарата.

Студентам полезно предложить типовые задачи на линейные операции над векторами, построение и анализ n-мерных арифметических пространств, выявление линейной зависимости и линейной независимости системы векторов, вычисление скалярного произведения векторов. Важно добиться понимания отличий частного от общего, опорного от базисных решений системы уравнений, понимание методов решения и геометрической интерпретации систем уравнений и неравенств.

Для обсуждения в электронной образовательной среде можно вынести такие вопросы как элементарные преобразования матрицы и нахождение ранга матрицы, нахождение координат произвольного вектора в заданном базисе с акцентом на типовые ошибки, наиболее часто встречающихся в работах студентов экономического бакалавриата.

Раздел 2. Различные постановки и различные формы записи задачи линейного программирования, геометрический смысл задачи линейного программирования для реализачии геометрического (графического) метода решения задачи линейного программирования. Самостоятельный подбор экономических ситуаций, приводящих к задачам линейного программирования в различных формах записи. При этом студентам экономического бакалавриата можно предложить как адаптацию уже сконструированных типовых экономических ситуаций, так и конструирование экономических ситуаций «с нуля». Различные постановки и различные формы записи задачи линейного программирования можно рекомендовать представить в виде иерархической структуры. В процессе подготовки студентов к практическим занятиям необходимо предусмотреть отработку процесса перехода от одной формы записи владение различными формами записи задачи линейного программирования.

Среди типовых ошибок, допускаемых студентами экономического бакалавриата при прохождении технологических диагностик, является неверное определение множества допустимых решений, непонимание смысла целевой функции, ошибки при введении переменных на первом этапе формализации экономической ситуации (содержательный смысл, количество, тип переменных), неверное построение семейства линий уровня, ошибки в процессе графического и аналитического определения координат угловой точки.

При работе над домашними заданиями необходимо обеспечить реализацию всех этапов графического метода решения задачи линейного программирования, а также формирование навыков сопоставления различных форм записи задачи линейного программирования с основными типами социально-экономических ситуаций. Выполнение типовых расчетов по содержанию данного раздела должно быть направлено на то, чтобы студенты экономического бакалавриата умели решать задачи линейного программирования геометрическим методом, владели навыками проведения содержательного анализа полученного решения, понимали совокупность неизвестных переменных величин как вектор, различали стандартную (симметричную) задачу линейного программирования и каноническую (основную) задача линейного программирования.

Работа с тестовым материалом, представленным в корпоративной системе LMS РЭУ им. Г. В. Плеханова направлено на развитие умений правильного нахождения точки оптимума при решении задачи линейного программирования с учётом направление оптимизации, умение давать содержательную интерпретацию найденному оптимальному плану с 
последовательным вычислением экстремального значения целевой функции запятая в соответствующие цели анализируемой социально-экономической ситуации. Кроме того, студентам можно предложить самостоятельную работу с различными многоугольниками областями, образующими множества допустимых решений.

Раздел 3. Симплекс-метод как итераџионная процедура последовательного улучшения начального допустимого решения. Представление в виде иерархической структуры алгоритма решения задачи линейного программирования симплекс-методом и алгоритма приведения задачи линейного программирования к задаче с искусственным базисом позволяет добиться более глубокого понимания студентами экономического бакалавриата понимания сущности опорного и оптимального плана задачи, владение всеми этапами реализации алгоритма решения задачи линейного программирования симплекс-методом.

В рамках подготовка студентов к практическим занятиям необходимо акцентировать внимание на приёмах построения начальной симплекс таблицы, приемах определения разрешающего генерального элементы с симплекс таблицы, приемах перехода от начальной симплекс таблицы к последующей симплекс таблицы. При работе над домашними заданиями в виде отдельных прикладных задач социально-экономической тематики необходимо развивать умение применять критерий оптимальности при работе по симплекс методу, умение различать задачи на симплекс метод с естественным базисом и задачи на симплекс методом с искусственным базисом в скобках в скобках предполагающим использование м метода).

Для обеспечения успешного прохождения студентами технологических диагностик по данному разделу необходимо предусмотреть достаточное количество заданий различного уровня сложности не только на реализацию симплекс метода, но и на введение искусственных переменных с целью получения расширенной задачи линейного программирования, нахождение начального опорного плана расширенной задачи и использование симплекс метода последовательного исключения искусственных переменных.

Раздел 4. «Элементы теории двойственности в линейном программировании». Для качественного усвоения теоретического содержания четвертого раздела следует уделить достаточное внимание приёмам построения задачи двойственный к заданной задаче линейного программирования, обращая внимание студентов экономического бакалавриата на понимание взаимосвязи пары взаимно двойственных задач. Работа над домашними заданиями в виде отдельных прикладных задач социально-экономической тематики и типовых расчётов должна обеспечить навыки применения теорем двойственности при анализе социальноэкономических ситуаций (теорема оптимальности Канторовича, первая и вторая теоремы двойственности).

Необходимо предусмотреть достаточное количество задач для самостоятельной работы на формирование приемов экономического анализа, основанного на свойстве двойственных оценок, выработки навыков построения задачи двойственный к заданной, определение оптимального решения двойственной задачи по предварительно найденному оптимальному решению исходной задачи линейного программирования и осуществление экономикоматематического анализа задачи линейного программирования на основе элементов теории двойственности.

Раздел 5. «Транспортная задача и метод потенциалов для нахождения оптимального решения транспортных задач». Для представления в виде иерархических структур студентам экономического бакалавра целесообразно предложить открытую и закрытую транспортные модели, а также различные методы исследования транспортных моделей (приводящих как к допустимым планам, так и к оптимальному плану). При самостоятельном разборе типовых задач данного раздела необходимо добиться понимания опорного плана транспортной модели, развития умения применять всех этапов метода потенциалов. Вопросы для обсуждения в цифровой образовательной среде должны влачить такие понятия, как цикл пересчета и особенности применения свойства цикла пересчета в процессе исследования транспортных моделей. При составлении типовых расчётов необходимо предусмотреть процессе самостоятельного перехода от словесной формулировки транспортной задачи к её табличному 
виду. Технологическая диагностика по содержанию данного раздела должна охватывать следующие вопросы:

- формализация различных транспортных моделей;

- преобразовывайте открытой транспортной модели в закрытую;

- определение опорного плана различными методами;

- осуществление проверки оптимальности плана найденного плана по методу потенциалов;

- самостоятельный выбор и реализация цикла пересчета;

- самостоятельное нахождение оптимального плана и выявление его содержательного смысла.

Обратим внимание, что при организации самостоятельной работы студентов экономического бакалавриата необходимо предусмотреть как самостоятельное решение в традиционном смысле, так и его реализацию в цифровом инструментальном средстве (например, WolframAlpha) и сравнение полученных результатов, обеспечить понимание студентами экономического бакалавриата механизмов работы цифровых инструментальных средств и их ограничений.

Представленные содержательно-методические особенности организации самостоятельной работы при изучении математических дисциплин в высшей экономической в виде шести целей и пяти форм организации самостоятельной работы студентов экономического бакалавриата, конкретизированных на уровне профессионально значимой учебной темы «Линейное программирование» позволяют учитывать дидактические возможности новых цифровых инструментальных средств и цифровых технологий, реализовывать необходимую прикладную направленность обучения математическим дисциплинам в высшей экономической школе.

\section{Список литературы}

1. Власов Д. А., Синчуков А. В. Новое содержание прикладной математической подготовки бакалавра // Преподаватель XXI век. 2013. № 1-1. С. 71-79.

2. Зададаев С. А. Цифровое расширение преподавания базовой математики // Современная математика и концепции инновационного математического образования. 2018. T. 5. № 1. C. 308-314.

3. Карасев П. А., Чайковская Л. А. Совершенствование программ высшего образования в контексте современных требований рынков образовательных услуг и профессионального сообщества // Экономика и управление: проблемы, решения. 2017. T. 3. № 2. C. 3-9.

4. Клещева Н. А., Петрук Г. В. Разработка педагогического сопровождения процесса формирования профессионально важных качеств экономиста-менеджера в системе самостоятельной работы студентов // Фундаментальные исследования. 2011. № 8-3. C. 524-527.

5. Липагина Л. В. Проблемы изучения будущими экономистами математических дисциплин в реалиях цифрового образования // Современная математика и концепции инновационного математического образования. 2018. Т. 5. № 1. С. 347-352.

6. Мастяева И. Н., Горемыкина Г. И. Методы оптимальных решений. М.: КУРС, 2016. $384 \mathrm{c}$.

7. Матвиенко Е. Н. Организационно-педагогические условия эффективности самостоятельной работы студентов-экономистов // Вестник Сибирского государственного университета путей сообщения. 2014. № 31. С. 179-183.

8. Мелехина Т. Л., Поздеева С. Н. Приемы вовлеченности в обучение студентов различных уровней подготовки при изучении математических дисциплин / Новые технологии высшей школы. Наука, техника, педагогика: материалы Всероссийской научно-практической конференции. 2020. С. 333-336. 
9. Монахов В. М. Введение в теорию педагогических технологий. Волгоград, Перемена, 2006. $365 \mathrm{c}$.

10. Пантина И. В., Синчуков А.В. Вычислительная математика: учебник. М.: Синергия, 2012. $176 \mathrm{c}$.

11. Смирнов Е. И., Трофимец Е. Н. Проектирование информационно-аналитических технологий обучения студентов-экономистов // Ярославский педагогический вестник. 2010. T. 2. № 2. С. 137.

12. Сухорукова И. В., Чистякова Н. А. Методические подходы преподавания актуарной математики //Актуальные проблемы преподавания математики в техническом вузе. 2019. № 7. C. 310-314.

13. Тихомиров Н. П., Тихомирова Т. М. Теория риска: учебник для студентов вузов, обучающихся по экономическим специальностям. М.: ЮНИТИ-ДАНА, 2020. 308 с.

14. Феклин В. Г. Использование LMS Moodle для создания электронного математического курса // Современная математика и концепции инновационного математического образования. 2014. Т. 1. № 1. С. 233-240.

15. Хуторской А. В. Педагогические основания диагностики и оценки компетентностных результатов обучения // Известия Волгоградского государственного педагогического университета. 2013. № 5 (80). С. 7-15.

\title{
GOALS AND FORMS OF ORGANIZING THE INDEPENDENT WORK OF BACHELORS OF ECONOMICS IN THE STUDY OF MATHEMATICAL DISCIPLINES
}

\author{
D.A. Vlasov $\mid$ Plekhanov Russian University of Economics \\ PhD (Pedagogy), associate professor \\ DAV495@gmail.com \\ Moscow
}

\begin{abstract}
The focus of the article is the methodological features of organizing the independent work of bachelors of economics in the study of mathematical disciplines related to the development of the system of independent work of students of an economic bachelor's degree. Six goals of independent work of students of an economic baccalaureate are presented, allowing to focus on development of innovative components of professional activity of the future economist in conditions of digitalization. Special attention is paid to the forms of independent work of students of an economic bachelor's degree without the direct participation of teachers of mathematical disciplines.
\end{abstract}

Keywords: independent work, mathematical training, digitalization, higher economic school, digital environment, competence, bachelor of economics.

\section{References}

1. Feklin, V. G. (2014). Ispol'zovanie LMS Moodle dlya sozdaniya elektronnogo matematicheskogo kursa [Using LMS Moodle to create an electronic mathematical course]. Sovremennaya matematika $i$ koncepcii innovacionnogo matematicheskogo obrazovaniya [Modern mathematics and concepts of innovative mathematical education], 1(1), 233-240. (In Russ., abstract in Eng.)

2. Karasev, P. A., Tchaikovsky, L. A. (2017). Sovershenstvovanie programm vysshego obrazovaniya $\mathrm{v}$ kontekste sovremennyh trebovanij rynkov obrazovatel'nyh uslug i 
professional'nogo soobshchestva [ Improving higher education programs in the context of modern requirements of educational services markets and the professional community]. Ekonomika i upravlenie: problemy, resheniya [Economics and management: problems, solutions]. 3 (2), 3-9. (In Russ., abstract in Eng.)

3. Khutorskoy, A. V. (2013). Pedagogicheskie osnovaniya diagnostiki i ocenki kompetentnostnyh rezul'tatov obucheniya [Pedagogical bases of diagnostics and assessment of competence-based learning outcomes]. Izvestiya Volgogradskogo gosudarstvennogo pedagogicheskogo universiteta [Proceedings of the Volgograd State Pedagogical University]. 5 (80), 7-15. (In Russ., abstract in Eng.)

4. Kleshcheva, N. A., Petruk, G. V. (2011). Razrabotka pedagogicheskogo soprovozhdeniya processa formirovaniya professional'no vazhnyh kachestv ekonomista-menedzhera v sisteme samostoyatel'noj raboty studentov [Development of pedagogical support for the process of forming professionally important qualities of an economist-manager in the system of independent work of students]. Fundamental'nye issledovaniya [Fundamental research], 8-3, 524-527. (In Russ., abstract in Eng.)

5. Lipagina, L. V. (2018). Problemy izucheniya budushchimi ekonomistami matematicheskih disciplin $\mathrm{v}$ realiyah cifrovogo obrazovaniya [Problems of studying mathematical disciplines by future economists in the realities of digital education]. Sovremennaya matematika $i$ koncepcii innovacionnogo matematicheskogo obrazovaniya [Modern mathematics and concepts of innovative mathematical education], 5(1), 347-352. (In Russ., abstract in Eng.)

6. Mastyaeva, I. N., Goremykina, G. I. (2016). Metody optimal'nyh reshenij [Methods of optimal solutions]. Moscow: KURS. (In Russ.)

7. Matvienko, E. N. (2014). Organizacionno-pedagogicheskie usloviya effektivnosti samostoyatel'noj raboty studentov-ekonomistov [Organizational and pedagogical conditions for the effectiveness of independent work of students-economists]. Vestnik Sibirskogo gosudarstvennogo universiteta putej soobshcheniya [Bulletin of the Siberian State University of Railway Transport], 31, 179-183. (In Russ., abstract in Eng.)

8. Melekhina, T. L., Pozdeyeva, S. N. (2020). Priemy vovlechennosti v obuchenie studentov razlichnyh urovnej podgotovki pri izuchenii matematicheskih disciplin [Methods of involvement in teaching students of various levels of training in the study of mathematical disciplines]. Novye tekhnologii vysshej shkoly. Nauka, tekhnika, pedagogika: materialy Vserossijskoj nauchno-prakticheskoj konferencii [New technologies of higher school. Science, technology, pedagogy. Materials of the All-Russian scientific and Practical Conference], 333-336. (In Russ.)

9. Monakhov, V. M. (2006). Vvedenie v teoriyu pedagogicheskih tekhnologij [Introduction to the theory of pedagogical technologies]. Volgograd, Peremena. (In Russ.)

10. Pantina, I. V., Sinchukov, A.V. (2012). Vychislitel'naya matematika [Computational mathematics]. Moscow: Synergy. (In Russ.)

11. Smirnov, E. I., Trofimets, E. N. (2010). Proektirovanie informacionno-analiticheskih tekhnologij obucheniya studentov-ekonomistov [Designing information and analytical technologies for teaching economics students]. Yaroslavskij pedagogicheskij vestnik [Yaroslavl Pedagogical Bulletin], 2(2), 137. (In Russ., abstract in Eng.)

12. Sukhorukova, I. V., Chistyakova, N. A. (2019). Metodicheskie podhody prepodavaniya aktuarnoj matematiki [Methodological approaches to teaching actuarial mathematics]. Aktual'nye problemy prepodavaniya matematiki $v$ tekhnicheskom vuze [Actual problems of teaching mathematics in a technical university], 7, 310-314. (In Russ., abstract in Eng.)

13. Tikhomirov, N. P., Tikhomirova, T. M. (2020). Teoriya riska [Risk theory]. Moscow: UNITY-DANA. (In Russ.)

14. Vlasov, D. A., Sinchukov, A. V. (2013). Novoe soderzhanie prikladnoj matematicheskoj podgotovki bakalavra [New content of applied mathematical bachelor's training]. Prepodavatel' XXI vek [Teacher XXI century], 1-1, 71-79. (In Russ., abstract in Eng.) 
15. Zadadaev, S. A. (2018). Cifrovoe rasshirenie prepodavaniya bazovoj matematiki [Digital extension of teaching basic mathematics]. Sovremennaya matematika $i$ koncepcii innovacionnogo matematicheskogo obrazovaniya [Modern mathematics and concepts of innovative mathematical education], 5(1), 308-314. (In Russ., abstract in Eng.)

DOI: $10.24888 / 2500-1957-2021-3-29-35$

\title{
УДК \\ 372.851 СТОХАСТИЧЕСКОГО МИРОВОЗЗРЕНИЯ СТАРШЕКЛАССНИКОВ В ОБЩЕОБРАЗОВАТЕЛЬНОЙ ШКОЛЕ
}

\author{
Лыкова Ксения Геннадьевна $\mid$ Елецкий государственный университет \\ аспирант им. И.А. Бунина \\ ksli1024@mail.ru \\ г. Елец
}

Аннотация. Изучение элементов стохастики в школе знакомит учащихся с
вероятностно-статистическими методами, которые
исследованию изменчивости и сложности политических, экономических,
общественных процессов. Прикладные возможности вероятностно-
статистических методов, осваиваемые школьниками, обуславливают формирование у них «нового мировоззрения». Б.В. Гнеденко рассматривая понятие «статистическое мышление», причислял к нему качества, характеризующие «новое мировоззрение». Так, к характерным чертам «нового мировоззрения» им были отнесены: «Понимание того, что в мире случайного есть свои закономерности; умение, пользуясь простейшими из этих закономерностей моделировать случайные явления и прогнозировать их исходы; умение и привычка за обобщающими вероятностными понятиями видеть их статистическую природу; умение анализировать большие совокупности с помощью вероятностно-статистических законов и содержательно интерпретировать полученные результаты» [1]. Учащиеся, выполняя на уроках математики оценку вероятностной природы явлений окружающей действительности и применяя её результаты в дальнейшем, приобретают мировоззренческий опыт. Специфика мировоззрения заключается в том, что оно выражает характер мыслительной деятельности, отношение человека к миру, проявляясь в его практической деятельности. В связи с чем проблема развития стохастического мировоззрения учащихся 10-11 классов является крайне актуальной. Особенностью стохастического мировоззрения является формирование у старшеклассников положительного отношения к случайностям, умений устанавливать межпредметные связи и прогнозировать поведение объектов в окружающей среде. Вследствие планомерного изучения случайных событий, случайных величин с последующим установлением межпредметных связей, у учащихся выстроится миропонимание, произойдет переосмысление механизмов функционирования мира. Стохастическое мировоззрение формируется на основе получаемого индивидуального опыта и набора специфических стохастических знаний, а под воздействием стохастического мировоззрения у старшеклассников будет формироваться объективный взгляд на мир и жизненная позиция. 